\title{
The 2011 Tuscaloosa Tornado: Integration of Pediatric Disaster Services into Regional Systems of Care
}

\author{
Robert K. Kanter, MD ${ }^{1,2}$
}

\begin{abstract}
Objective To empirically describe the integration of pediatric disaster services into regional systems of care after the April 27, 2011, tornado in Tuscaloosa, Alabama, a community with no pediatric emergency department or pediatric intensive care unit and few pediatric subspecialists.

Study design Data were obtained in interviews with key informants including professional staff and managers from public health and emergency management agencies, prehospital emergency medical services, fire departments, hospital nurses, physicians, and the trauma program coordinator.

Results A single hospital in Tuscaloosa served 800 patients on the night of the tornado. More than 100 of these patients were children, including more than 20 with critical injuries. Many children were unaccompanied and unidentified on arrival. Resuscitation and stabilization were performed by nonpediatric prehospital and emergency department staff. More than $\mathbf{2 0}$ children were secondarily transported to the nearest children's hospital an hour's drive away under the care of nonpediatric local emergency medical services providers. No preventable adverse events were identified in the resuscitation and secondary transport phases of care. Stockpiled supplies and equipment were adequate to serve the needs of the disaster victims, including the children.

Conclusion Essential aspects of preparation include pediatric-specific clinical skills, supplies and equipment, operational disaster plans, and interagency practice embedded in everyday work. Opportunities for improvement identified include more timely response to warnings, improved practices for identifying unaccompanied children, and enhanced child safety in shelters. Successful responses depended on integration of pediatric services into regional systems of care. (J Pediatr 2012; $\mathbf{0} \mathbf{\square}-\mathbf{\square})$.
\end{abstract}

isasters may injure children far from a pediatric hospital, and disaster surges of children can overwhelm pediatric facilities. Thus, the need to integrate pediatric disaster services into regional systems of care has been recognized. ${ }^{1-3}$ Little experience with pediatric care in major disasters has been reported, and commentators have called for better empirical data to guide preparedness. ${ }^{4,5}$

The severe tornado occurring in Tuscaloosa, Alabama on April 27, 2011, provides a case study to empirically examine pediatric-specific services and their integration into regional systems of care after a recent major disaster. In a community with no pediatric emergency department (ED) or pediatric intensive care unit (ICU) facing one of the largest ED disaster surges at a single hospital in US history, the successes provide a model for other regions, and the lessons learned suggest improvement opportunities for all.

\section{Methods}

Tuscaloosa is a city of 90468 people in which pediatricians, general practitioners, and a limited number of pediatric subspecialists provide ambulatory care for children. ${ }^{6}$ Pediatric acute care subspecialists in the community are limited to neonatologists. Tuscaloosa is served by skilled and experienced prehospital providers, a general ED, and an adult ICU at a single hospital. Prehospital care is usually provided according to statewide emergency medical services (EMS) protocols, with some, but not all, advanced life support interventions requiring medical control phone or radio authorization. The hospital is the 538-bed DCH Regional Medical Center, whose services include the regional trauma center and an ED serving an average of 185 patients per day. A 20-bed pediatric inpatient area with an average census of 8 serves common low-risk medical, traumatic, and surgical conditions. Because the regional medical center lacks a pediatric ED or ICU, critically ill or injured children are usually transferred from Tuscaloosa to the nearest children's hospital in Birmingham, an hour's drive away, under the medical control of pediatric transport coordinators at the children's hospital.

Pediatric disaster services and regional systems of care after the April 27, 2011, tornado were explored in key informant interviews, conducted between June and

\footnotetext{
ED Emergency department

EMS Emergency medical services

ICU Intensive care unit
}

From the ${ }^{1}$ Division of Pediatric Critical Care Medicine,

Department of Pediatrics, SUNY Upstate Medical

University, Syracuse, NY; and ${ }^{2}$ National Center for

Disaster Preparedness, Mailman School of Public

Health, Columbia University, New York, NY

The author declares no conflicts of interest.

0022-3476/\$ - see front matter. Copyright $\odot 2012$ Mosby Inc. All rights reserved. 10.1016/j.jpeds.2012.02.016 
August 2011. Informants included professional staff and managers from state and local public health and emergency management agencies, prehospital EMS, fire departments, hospital nursing and physician staff, and the regional trauma program coordinator. A total of 19 interviews were conducted, 14 individually and 5 in groups, involving a total of 50 informants. All interviews were conducted face to face except for one phone interview.

The semistructured interviews began with open-ended questions regarding local disaster mitigation, preparation, and responses to the April 27 tornado (Appendix; available at www.jpeds.com). Clarifying details and examples were sought in the interviews, as well as from other cited publicly available sources. Notes were taken during all interviews. Interviews were recorded with the consent of the informants. Notes and recordings were analyzed to identify themes pertaining to integration of pediatric services into regional systems. This study of professionals' roles and observations was considered exempt from review by the Institutional Review Board for the Protection of Human Subjects at SUNY Upstate Medical University.

\section{Results}

An F4 tornado passed across Tuscaloosa at $5 \mathrm{PM}$ on Wednesday April 27, 2011. The path of severe damage across residential and commercial neighborhoods ranged from 0.5 to 1 mile wide and was more than 5 miles long, damaging or destroying more than 5700 structures in an area directly affecting 13700 people. $^{7}$ Forty-six deaths occurred, with a mortality rate of 3.4 deaths per 1000 population in the directly affected area. ${ }^{8,9}$ As reflected by fatal injuries, children were involved in proportion to their numbers in the population. Eight deaths occurred in children aged $<18$ years ( $17 \%$ of all deaths), ${ }^{9}$ and children aged $0-17$ years account for $17.4 \%$ of Tuscaloosa's population. ${ }^{6}$

\section{Response, First Hour}

The entire severe damage zone was initially impassable to vehicles. Several severely injured children were among the first victims found, including some who had been playing in a playground when the storm hit. In the rubble of destroyed homes, some fatally injured adults were found lying over children in successful efforts to protect them.

During the first hour after the storm, fire departments set up command posts and EMS staffed triage and treatment centers at the edge of the severe damage zone. Although the damaged area remained inaccessible to vehicles, victims were carried by hand, pulled on mattresses, or rolled on wheelbarrows to the triage and treatment areas. Care in the field was limited to first aid and immediate life-saving interventions. Occasionally paramedics considered advanced life support treatment, such as needle decompression of a suspected tension pneumothorax in a child, balancing the urgency of field interventions against the short ride to the hospital in a setting of overwhelming needs. Private vehicles transported some injured patients to the hospital before an adequate number of ambulances were available.

Later during the first hour, the Tuscaloosa Department of Transportation began clearing main roads into the severe damage zone. As a result, all-terrain vehicles, small pickup trucks, and later fire trucks and ambulances could carry rescue and medical equipment into and transport patients out of the heavily damaged area.

The tornado passed within 2 city blocks of DCH Regional Medical Center. Inpatients and staff had been moved to internal locations away from windows just before the storm hit. A few windows were blown out, power was lost and then immediately restored by generators, but the hospital sustained no other direct damage. The hospital initiated emergency incident command procedures. Clinical staff were assigned to their usual work locations or alternate areas where they had previously received cross-training. Emergency physicians, anesthesiologists, and general and orthopedic surgeons were assigned to care for critically injured persons. Neonatologists assisted with advanced life support of infants. Primary care pediatricians were assigned to care for children after initial resuscitation and for those less severely injured. Family medicine and internal medicine practitioners cared for adults with moderate and milder injuries. Radiologists interpreted images immediately.

\section{Response, First Night}

All patients from Tuscaloosa needing ED care were transported to DCH Regional Medical Center because of the longer distance to other facilities, and because a continuing tornado threat in the region interfered with primary transport elsewhere. Given the overwhelming workload in the field and at the hospital, hospital clinical leaders instructed EMS staff to provide all necessary prehospital emergency care according to statewide EMS protocols, without phone or radio medical control. At the hospital, premade hospital administrative packages to identify and register 450 patients were used up in less than 4 hours. Subsequently, the ED used EMS disaster patient identification tags. With so many patients moving through the ED, it became difficult to track patients and match reports of critical diagnostic tests with the location of patients requiring immediate interventions.

Dozens of unaccompanied children arrived at the ED, identifiable only by the location from which they were transported. Some were too young or severely injured to provide information. Others, because of fear and stress, simply refused to speak.

Along with a large number of adults, more than 20 children with critical traumatic brain, chest, or abdominal injuries required endotracheal intubation in the ED. Intubation, chest tube placement, and vascular access, including central venous catheter insertion, in children were performed by emergency physicians, anesthesiologists, and surgeons, and some infants were intubated by neonatologists. Two children required immediate craniotomy, and other pediatric surgical interventions were delayed. Critically injured children were kept in the ED, receiving 1:1 nursing 
or physician care for the first several hours, including airway maintenance, ventilation, and fluid and analgesic management, until secondary transport could be arranged. Children with less severe injuries were admitted to the pediatric floor at DCH or discharged. Of the approximately 800 patients receiving care in the ED during the night of the storm, more than 100 were children.

Phone discussions occurred in the first hours between physicians in Tuscaloosa and Children's Hospital of Alabama in Birmingham regarding the care of critically injured children and plans for transfer. The idea of sending pediatric staff from Birmingham to provide care at Tuscaloosa was rejected, because this would have depleted the staff needed to serve statewide pediatric referrals at Children's Hospital. Instead, Children's Hospital instructed DCH to secondarily transport critically injured children after stabilization, under the care of local Tuscaloosa staff. No further attempt was made to use pediatric critical care transport teams, because insufficient staff and vehicles at Children's Hospital would impose long delays on the mass evacuation. More than 20 critically injured children were transferred later that night from $\mathrm{DCH}$ in Tuscaloosa to Children's Hospital of Alabama in Birmingham. Secondary transport care was provided by nonpediatric EMS staff from the Tuscaloosa area, using statewide EMS advanced life support protocols without real-time radio medical control because of the overwhelming workload of hospital staff. Most interhospital transports were performed by ground ambulance, because weather conditions limited helicopter flights. Subsequent reviews conducted at Tuscaloosa and Birmingham concluded that pediatric resuscitations and interhospital transports were accomplished without a single clinically significant preventable adverse event (death or major physiological deterioration).

In addition to transfers from DCH in Tuscaloosa, Children's Hospital of Alabama in Birmingham evaluated a total of 60 pediatric trauma victims injured by multiple tornadoes across 13 counties, 39 of whom required admission. ${ }^{10} \mathrm{~A}$ relatively low patient census at the time of the disaster allowed this large number of admissions. Contingency plans were considered to request National Guard assistance in transporting children exceeding the surge capacity of Children's Hospital to pediatric hospitals in adjacent regions. Transport outside the region was not necessary, however.

Attempts to identify unaccompanied children continued at Children's Hospital. In some cases, digital photos were sent electronically for viewing and identification by family members at remote locations over the next several days.

Although the tornado resulted in historically large pediatric and adult ED surges, statewide stockpiles of equipment and supplies strategically located near DCH Regional Medical Center and Children's Hospital of Alabama were sufficient to provide all life-saving interventions. In Tuscaloosa, trailers containing stockpiled items arrived at the hospital within the first four hours after the storm. In particular, supplies of pediatric endotracheal tubes were sufficient, although occasionally smaller available endotracheal tubes had to be substituted for unavailable sizes. Disaster stockpiles of ventilators stored at Children's Hospital were used to supplement the usual hospital inventory. In addition to life support equipment, informants emphasized the importance of adequate supplies of diapers and infant formula for a busy ED or shelter serving many infants.

On the night of the storm, the Red Cross opened a large public shelter accommodating 600 individuals in Tuscaloosa. Buses shuttled large numbers from triage treatment centers and from the hospital ED to the shelter. Although some managers anticipated security problems in shelters serving families with children along with an unscreened adult population, informants were not aware of any adverse incidents.

\section{Response, Next Several Days}

Although individuals with chronic medical conditions were encouraged to relocate from homes lacking electrical power, some families refused. One technology-assisted child was supported at home with rechargeable batteries, and later with a donated generator.

\section{Early Mitigation and Preparedness}

Successes in emergency responses are best understood in the context of previous efforts to reduce risk and prepare for emergencies. Early preparation was informed by the high frequency of severe weather events in the region. Tuscaloosa had well-developed plans for tornadoes, as well as for sheltering populations after Gulf Coast evacuations ahead of hurricanes. Interagency practice for responses was embedded in everyday work as a result of frequent severe weather warnings. State funds provided the strategically located equipment and supply stockpiles that enabled immediate responses to ED surges on the night of the storm. In addition, federal EMS for Children grants had been used to purchase pediatric-specific equipment used in resuscitation of dozens of critically injured children. Although very few pediatric acute care subspecialists were available in Tuscaloosa, many hospital and EMS staff had training in pediatric advanced life support skills.

Emergency preparedness in Tuscaloosa is also maintained by practice associated with the periodic doubling of the community population size on 7 autumn weekends each year during home football games at the University of Alabama. In addition to anticipating security and communications issues, EMS must serve dozens of illnesses among spectators.

Communication among emergency managers during the first night and subsequent days was reliably conducted using the SouthernLINC cell phone network provided by the power utility company throughout Alabama. The reliability of these telephones depends on rapid deployment of mobile equipment to replace damaged towers and a priority system that connects emergency managers' calls before those of other users. In contrast, commercial cell phones serving the public failed for several days after the storm.

In addition to plans and practice, many informants emphasized that successful responses depend on resourceful leaders and staff adapting to unforeseen circumstances based on their training and experience. Interagency coordination 
depends on frequent everyday non-disaster professional interactions. Many informants also emphasized the importance of trust and familiarity among leaders based on longstanding personal relationships, citing membership in the same churches and other community organizations.

\section{Last Day Mitigation and Preparedness}

Based on regional predictions of tornado risk exceeding 95\%, ${ }^{11}$ local preparations on April 27 included maximizing the number of ambulances in service and reassigning EMS staff from administrative to clinical duties. A mobile support and repair vehicle activated earlier in the day was important for keeping ambulances in service after the storm. Just-intime training of EMS staff emphasized attention to basic tasks.

Many schools and businesses throughout Alabama had been closed on April 27 in anticipation of severe weather. Although the Tuscaloosa tornado passed through the community late in the afternoon hours after schools would have been dismissed, the early closure of some businesses might have reduced the amount of rush hour traffic vulnerable to the storm. Risk reduction also was provided by lastminute tornado warning sirens and televised warnings of the approaching tornado.

\section{Discussion}

Responses to the Tuscaloosa tornado of April 27, 2011, provide an example of highly effective services for children integrated into regional systems of disaster care. Essential preparation included pediatric-specific clinical skills, pediatric supplies and equipment, general operational disaster planning that included interagency practice embedded in everyday work, and experienced resourceful leaders and staff.

Several pediatric-specific opportunities for improvement were apparent. First, although warnings of the approaching tornado were accurate and timely, some in the community failed to either receive or heed the warnings. Children were overrepresented among the population outdoors at the time of the tornado, and they suffered a high rate of fatal injuries. Thus, risk communications targeting children warrant improvement. Second, practices for identifying and tracking unaccompanied children in disasters could be strengthened. Third, security procedures for shelters serving children must account for unscreened adults sharing the same facility.

The value of interagency practice in nondisaster everyday work has been emphasized recently. ${ }^{12}$ For example, large sporting events requiring community-wide coordination can be an occasion to improve incident command proficiency and interagency collaboration. Although an inventory of pediatric-specific disaster stockpiles was not available from key informants in Alabama, detailed recommendations on supplies and equipment have been published recently. ${ }^{13}$ In all regions, strategic locations of stockpiles must be tailored to local circumstances if the supplies are to be immediately available. The decision to provide interhospital mass evacua- tion without pediatric critical care transport teams was consistent with results of a discrete event simulation. ${ }^{14}$ In this case, the advantage of more rapid transport by local nonpediatric EMS teams was not compromised by preventable adverse events. The local direction of disaster responses, supported by statewide resources, was consistent with the national framework for disaster operations. ${ }^{15}$

This study based on key informant interviews reports quantitative and semiquantitative observations, as well as qualitative insights provided by expert participants in the disaster response. The design does not permit a quantitative analysis of hypotheses. Whether qualitative or quantitative, lessons learned in a single historical event cannot provide complete guidance for responses to future emergencies. Nevertheless, this report adds empirical experience to a sparse existing database regarding pediatric disaster responses and may contribute to planning in communities lacking pediatric acute care subspecialists. Successful responses to the Tuscaloosa tornado serve as a challenge to all of us. How many regions could match Alabama's performance?

I thank all the professional staff involved with responses to the Tuscaloosa tornado of April 27, 2011, whose assistance as key informants contributed to this study. The following individuals provided extensive information, as well as invaluable assistance in identifying other informants essential to conducting this study: Glenn Davis, Region 4 EMS Director, Tuscaloosa, AL; Andrew Lee, RN, Trauma Program Coordinator, DCH Regional Medical Center, Tuscaloosa, $A L$; Steve Baldwin, MD, Pediatric Emergency Medicine, Children's Hospital of Alabama, Birmingham, AL; Katherine Dixon Hert, EMSC Program Manager, Alabama Department of Public Health, Montgomery, AL; and Martha Gohlke, EMSC Program Coordinator, New York State Department of Health, Troy, NY.

Submitted for publication Nov 4, 2011; last revision received Feb 7, 2012; accepted Feb 13, 2012.

\section{References}

1. Kanter RK, Cooper A. Mass critical care: pediatric considerations in extending and rationing care in public health emergencies. Disaster Med Public Health Prep 2009;3:166-71.

2. Barfield WD, Krug SE, Kanter RK, Gausche-Hill M, Brantley MD, Chung S, et al. Neonatal and pediatric regionalized systems in pediatric emergency mass critical care. Pediatr Crit Care Med 2011;12: S128-34.

3. National Commission on Children and Disasters. 2010 report to the President and Congress. Available from: http://cybercemetery.unt.edu/ archive/nccd/20110427002908/http://www.childrenanddisasters.acf.hhs. gov/index.html. Accessed September 8, 2011.

4. Johnston C, Redlener I. Summary of issues demanding solutions before the next one. Pediatrics 2006;117:S458-60.

5. Yeager VA, Menachemi N, McCormick LC, Ginter PM. The nature of the public health emergency preparedness literature, 2000-2008: a quantitative analysis. J Public Health Manag Pract 2010;16:441-9.

6. United States Census Bureau. State and county quick facts-Tuscaloosa, Alabama. Available from: http://quickfacts.census.gov/qfd/states/01/ 0177256.htm. Accessed January 4, 2012.

7. Hanna J. Cable News Network. Tuscaloosa mayor: eyes back on tornado catastrophe. Available from: http://articles.cnn.com/2011-05-05/ us/alabama.tornadoes.mayor_1_mayor-walter-maddox-tuscaloosamayor-massive-tornado?_s=PM:US. Accessed August 1, 2011. 
8. Flanagan B. The Birmingham News. Three added to Tuscaloosa tornado death toll. Available from: http://blog.al.com/tuscaloosa/2011/07/three_ names_added_to_tuscaloos.html. Accessed August 1, 2011.

9. Reynolds B. Tuscaloosa News. City releases names of tornado victims. Available from: http://www.tuscaloosanews.com/article/20110501/ NEWS/110509969. Accessed July 14, 2011.

10. Children's Hospital of Alabama. Pediatric injury updates, $4 / 28 / 11$ and $4 /$ 29/11. Available from: http://www.childrensal.org/body.cfm?id=1454. Accessed August 15, 2011.

11. National Oceanic and Atmospheric Administration Storm Prediction Center, Norman, Oklahoma. Tornado watch 235 watch hazard probabilities. Available from: http://www.spc.noaa.gov/products/watch/ ww0235_prob.html. Accessed August 1, 2011.
12. McCarthy DM, Chiampas GT, Malik S, Cole K, Lindeman P, Adams JG. Enhancing community disaster resilience through mass sporting events. Disaster Med Public Health Prep 2011;5: 310-5.

13. Bohn D, Kanter RK, Burns J, Barfield WD, Kissoon N. Supplies and equipment for pediatric emergency mass critical care. Pediatr Crit Care Med 2011;12:S120-7.

14. Kanter RK. Regional variation in critical care evacuation needs for children after a mass casualty incident. Disaster Med Public Health Prep 2012. in press.

15. Federal Emergency Management Agency. National response framework. Available from: http://www.fema.gov/emergency/nrf/. Accessed September 8, 2011. 


\section{Appendix. Template for Semistructured Interviews}

What roles and services did your organization provide for children and for others?

What was the effect of the disaster on your organization's ability to function? Did your responses proceed according to plans?

Interactions among response organizations?

Sources of information and methods of communication during the response? What went well?

What did not go well?

What improvements and adjustments has your organization already made as a result of this experience?

Lessons learned for next time? Next community? 